investigating the relationship between sleep quality, HRQoL and possible presence of subclinical inflammation in paediatric patients with IBD are warranted.

\section{MODERATE-TO-VIGOROUS PHYSICAL ACTIVITY IS ASSOCIATED WITH INCREASED MINERAL BONE DENSITY IN CHILDREN WITH INFLAMMATORY BOWEL DISEASE}

Ivana Trivič ${ }^{\star}$, Sara Sila, Ana Tripalo Batoš, Zrinjka Mišak, Sanja Kolaček, Iva Hojsak. Referral Centre for Paediatric Gastroenterology and Nutrition, Children's Hospital Zagreb

\subsection{6/archdischild-2021-europaediatrics.225}

Inflammatory bowel disease (IBD) in children is associated with malnutrition and growth failure. Body composition alternations, such as bone mass deficits, decreased bone mineral density (BMD) and reductions in lean body mass (LBM) have been described. Physical activity (PA) plays an important role in normal growth and development. Moderate-to-vigorous PA (MVPA) has beneficial effects on muscle mass accrual and bone health. Data regarding PA amongst children and adolescents with IBD are scarce. The aim of our study was to evaluate the relationship between $\mathrm{PA}$ and body composition in children with IBD in remission.

A total of 33 paediatric IBD patients in remission (20 boys) aged $15.6 \pm 1.9$ years were included in the study (disease type: Crohn's disease (CD), $n=16$, ulcerative colitis (UC), $\mathrm{n}=15$, inflammatory bowel disease-unclassified (IBD-U, $n=2$ ). Total body less head (TLBH) dual energy X-ray absorptiometry (DXA) was used to measure BMD, expressed as age- and sex-based Z-scores, and to assess fat mass (FM) and LBM, expressed in grams and as age-, sex- and height-based Zscores. Patients wore a triaxial accelerometer for five consecutive days for objective PA quantification. Daily caloric intake was assessed using a three day food intake record.

Mean BMD Z-score was $-0.41 \pm 0.88$; a third of patients had reduced BMD. Mean FM was $15718 \pm 6367.2 \mathrm{~g}$; fat mass

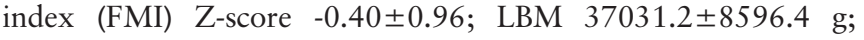
mean lean body mass index (LBMI) Z-score $-1.83 \pm 1.27$. On average, patients spent 38 minutes per day in MVPA. BMD Z-score positively correlated with body height (coef. 0.502, $\mathrm{p}=0.003$ ), body weight-for-age Z-score (coef. 0.742, $\mathrm{p}<0.001$ ), body mass index (BMI) $\mathrm{z}$-score (coef. 0.558, $\mathrm{p}=0.001)$. BMD Z-score positively correlated LBMI Z-score (coef. 0.758, p<0.001) and minutes spent in MVPA (coef. $0.513, \mathrm{p}=0.004)$. LBMI Z-score positively correlated with Zscore (coef. $0.758, \mathrm{p}<0.001$ and negatively correlated with cumulative corticosteroid dose $(\mathrm{mg} / \mathrm{kg} / \mathrm{y}$ ) (coef. -0.436 , $\mathrm{p}=0.011$ ). No differences regarding anthropometric and body composition parameters were observed between types of IBD. Female patients had statistically lower total LBM compared to male patients, but there was no difference in LBMI Z-scores. No correlation was found between daily caloric intake, daily protein, calcium, phosphorus and vitamin D intake and anthropometric, body composition and bone health parameters.

Positive correlation was found between BMD and lean body mass, as well as between BMD and minutes spent in MVPA. Intervention studies investigating a causal relationship between PA and favourable body composition in paediatric patients with IBD are warranted.

\section{METHEMOGLOBINEMIA IN 2 EXCLUSIVELY BREASTFED INFANTS WITH FOOD PROTEIN-INDUCED ENTEROCOLITIS SYNDROME}

Antonella Geljic*, Iva Hojsak. Children's Hospital Zagreb

10.1136/archdischild-2021-europaediatrics.226

Food protein-induced enterocolitis syndrome (FPIES) is a nonimmunoglobulin E (IgE)-mediated gastrointestinal food hypersensitivity of infancy, characterized by repetitive profuse vomiting, often in association with lethargy, pallor and diarrhea. It is most commonly caused by cow's milk protein (CMP) and soy. Breastfeeding is suggested to have a protective role, and FPIES to CMP in exclusively breastfed infants is extremely rare. We report 2 cases of FPIES to CMP in exclusively breastfed infants who both had methemoglobinemia.

The first patient, a 6-week old male exclusively breastfed infant, was transferred to our hospital due to the worsening of enterocolitis syndrome.

He presented with persistent bloody diarrhea up to 14 times per day, followed by vomiting and fever.

The second patient was an exclusively breastfed female infant who presented at the age of 2 months to our emergency department for persistent diarrhea, vomiting and anorexia without fever.

Both patients at admission were severely ill, pale, adynamic, somnolent and dehydrated. Laboratory findings showed metabolic acidosis, methemoglobinemia, anemia and hyponatremia. After laboratory work-up he was placed on antibiotics, but without significant improvement in clinical status and with persistent watery diarrhea. All laboratory work up was negative (radiologic findings, all cultures (stool, blood, cerebrospinal fluid, urine)), all performed metabolic and immunologic tests were negative.

Highest MHgb level was $5.7 \%$ in patient 1 and 9.1\% in patient 2. Due to severe diarrhea patients were paced on total parenteral nutrition and breastfeeding was stopped for the whole day. After that small quantities of elemental formula were slowly introduced. Symptoms improved and after several days breastfeeding was reintroduced with strict elimination of $\mathrm{CM}$ from mothers diet.

Specific IgE and skin prick test for milk was negative in both patients and atopy patch test was performed only in patient 2 and was negative. In both patient $\mathrm{CM}$ challenge test was performed at the age of 12 month without reaction; since then both patients tolerate milk.

FPIES in exclusively breastfed infants is extremely rare but should be taken into consideration in cases of unexplained severe enterocolitis. Transient methemoglobinemia may occur in such patients and usually does not require treatment.

\section{DYSAUTONOMIA IN CHILDREN WITH IRRITABLE BOWEL SYNDROME AND INFLAMMATORY BOWEL DISEASE}

Antonella Geljic*, Anamari Junakovic, Ivana Trivic, Sara Sila, Magdalena Krbot Skoric, Mario Habek, Iva Hojsak. Children's Hospital Zagreb

10.1136/archdischild-2021-europaediatrics.227

To evaluate the presence of autonomic nervous system abnormalities (ANS) in children with irritable bowel syndrome (IBS) 
and quiescence inflammatory bowel disease (IBD) comparing to controls.

Consecutive children with quiescence IBD, IBS and aged and sex matched healthy controls (HC) were referred for the evaluation of dysautonomia (IBD:

$\mathrm{N}=24$, mean age 15.7 yrs, 16 females; IBS: $\mathrm{N}=18$, mean age 14.8 yrs, 9 females; HC: $\mathrm{N}=18$, mean age 14.2 yrs, 9 females). Dysautonomia was evaluated subjectively with the Composite Autonomic Symptom Score (COMPASS 31), and objectively with the following autonomic tests: heart rate (HR) and blood pressure (BP) responses to the Valsalva maneuver, heart rate response to deep breathing (RSA), blood pressure response to passive tilt, and quantitative sudomotor axon reflex test (QSART). Additionally, heart rate variability (HRV) analysis was performed by Kubios HRV 2.2. Following HRV parameters were compared between the groups in supine and tilted positions: total power of low (LF) and high frequency domain components (HF), normalized HF (HFnu), low-to-high frequency ratio ( $\mathrm{LF} / \mathrm{HF})$, standard deviation of normal-to-normal intervals (SDNN) and percentage of successive RR intervals that differ by more than $50 \mathrm{~ms}$ (PNN50).

Children with IBS scored highest on COMPASS-31, followed by patients with IBD and HC (median 15.6, 8.7 and 2.3 , respectively, $\mathrm{p}<0.001$ ). Similar differences were observed in the orthostatic intolerance and gastrointestinal domains of the COMPASS-31. No differences between groups were observed in HR and BP responses to the Valsalva maneuver, RSA and BP response to passive tilt. Children with IBS had higher sweat volumes on proximal lower leg on QSART (median IBD 0.9, IBS 1.5, HC $0.8 \mu \mathrm{L} ; \mathrm{p}=0.039$ ).

There was no difference in the HRV parameters between groups. However, children with IBS had significantly higher drop in LF $(p=0.01)$ and SDNN

$(p=0.03)$ and lowest drop in PNN50 $(p=0.01)$ during tilt test compared to children with IBD and HC.

We found significant subjective and objective ANS abnormalities in children with IBS compared to children with IBD and $\mathrm{HC}$.

\section{INCIDENCE AND GEOGRAPHICAL VARIABILITY OF PEDIATRIC INFLAMMATORY BOWEL DISEASE IN CROATIA: DATA FROM THE CROATIAN NATIONAL REGISTRY}

Lana Ivković*, Iva Hojsak, Ivana Trivić, Sara Sila, Pero Hrabač, Vlatka Konjik, Irena SenečićČala, Goran Palčevski, Ranka Despot, Orjena Žaja, Sanja Kolaček. Children's Hospital Srebrnjak

\subsection{6/archdischild-2021-europaediatrics.228}

Primary aims of this study were to determine the annual incidence and geographic distribution of pediatric inflammatory bowel disease (IBD), respectively existence of north to south gradient in Croatia as well as baseline characteristics at the time of diagnosis. Secondary aim was to compare data on frequency and distribution of pediatric IBD in two consecutive years.

This is a prospective, cohort, multicenter observational study based on the data obtained from the newly established Croatian national registry for children with inflammatory bowel disease. Children and adolescents younger than 18 years diagnosed with IBD, according to revised Porto criteria, in time period between June 1st, 2016 and May 31st, 2017 were recruited. In order to validate obtained data and to monitor trends in the incidence of the disease, the data were also gathered for the period from June 1st, 2017 to May 31st, 2018.

In season 2016/2017, 51 new cases of pediatric IBD were identified; 19

(37.3\%) Crohn's disease (CD), 28 (54.9\%) ulcerative colitis (UC) and 8

(7.8\%) IBD unclassified (IBD-U). Male preponderance of all three types of the disease was noticed (CD 52.6\%, UC $60.7 \%$, IBD-U 75\%). The median age at diagnosis was 14.8 years; 15.3 for CD, 13.9 for UC, and 14.5 years for IBD-U. Female patients were slightly older than males, but without statistically significant difference $(p=0.279)$. With 723,552 children younger than 18 years in Croatia in 2017 , the annual incidence of pediatric IBD per 100,000 persons per year was 7.05 (2.62 for CD, 3.87 of UC and 0.55 for IBD-U). The lowest incidence was found in Dubrovnik-Neretva and SplitDalmatia County (4.50-4.85/100,000 children up to 18 years of age/year), and the highest was found in Meðimurje County (22.80/100,000 children up to 18 years of age/year). Existence of north to south gradient was observed with almost two times higher incidence in northern region compared with southern parts of the country $(8.38 / 100,000$ vs. $4.26 /$ 100,000).

Data from consecutive season (2017/2018) identified 50 new cases of pediatric IBD, with higher proportion of CD (44\%) compared to year before.

The incidence of pediatric IBD in Croatia is 7.05 per 100,000 persons younger than 18 years per year, with significant decreasing trend in incidences between northern and southern regions of the country.

\section{IBD PHENOTYPE ON DISEASE PRESENTATION AND EARLY DISEASE-COURSE IN PEDIATRIC PATIENTS IN CROATIA: DATA FROM THE CROATIAN NATIONAL REGISTRY}

Lana Ivković* ${ }^{*}$ Iva Hojsak, Ivana Trivić, Sara Sila, Pero Hrabač, Vlatka Konjik, Irena SenečićČala, Goran Palčevski, Ranka Despot, Orjena Žaja, Sanja Kolaček. Children's Hospital Srebrnjak, Zagreb, Croatia

10.1136/archdischild-2021-europaediatrics.229

Aims of this study were to determine the phenotype of pediatric inflammatory bowel disease (IBD) in patients in Croatia at the time of disease presentation, evaluate diagnostic and therapeutic approaches and investigate early disease-course in oneyear follow-up.

During a one-year time period (June 1st, 2016 to May 31st, 2017), children and adolescents younger than 18 years of age in Croatia with newly diagnosed IBD were prospectively recruited into Croatian national registry. Data on disease location and behavior (according to Paris classification), diagnostic evaluation and therapy used for inducing and maintaining remission were collected. One-year follow-up data examined relapse rates, as well as modification of medical therapy.

A total of 51 newly diagnosed patients were recruited: 19 (37.3\%) Crohn's disease (CD), 28 (54.9\%) ulcerative colitis (UC) and 4 (7.8\%) IBD - unclassified (IBD-U). Most common location in $\mathrm{CD}$ was ileocolonic (L3) disease in $52.6 \%$, followed by distal $1 / 3$ ileal disease (L1) in 31.6\%. 